\title{
THE UNIQUENESS OF A CERTAIN LINE INVOLUTION
}

\author{
C. R. WYLIE, JR.
}

In a forthcoming paper [1] ${ }^{1}$ on the classification of line involutions we pointed out that any line involution in $S_{3}$ with the property that a general line and its image do not intersect has associated with it, besides its order, $m$, and the order, $i$, of its invariant complex, two other important numerical characteristics which we called $k$ and $n$. The first of these, $k$, is the number of lines of an arbitrary pencil which meet their images. Recalling the well known mapping relation between the lines of $S_{3}$ and the points of a nonsingular $V_{4}^{2}$ in $S_{5}$, the second, $n$, is the order of the ruled surface formed by the rays which join the points of a general line of $V_{4}^{2}$ to their respective images. The four quantities, $m, i, k, n$, are not independent but satisfy the following relations [1]

$$
n-i=k, \quad n+i=m+1 .
$$

It is the purpose of the present note to establish the fact that there is a unique involution associated with the set $m=n=2, i=k=1$, namely the involution whose point equivalent on $V_{4}^{2}$ is defined by the transversals of a general line, $\lambda$, of $S_{5}$ and an $S_{3}$ contained in the $S_{4}$ which is tangent to $V_{4}^{2}$ at one of the two intersections of $\lambda$ and $V_{4}^{2}$ $[2,3]$. In constructing our proof we shall find it convenient to work exclusively in $S_{5}$ rather than in the three-dimensional space where the line involution is actually defined.

We shall begin by establishing the fact that the surface which is the image of a general plane of $V_{4}^{2}$ is of the second order. This cannot be inferred as obvious from the fact that the order of the transformation is two, because one may show by example that far from being self-evident, it is not even true that the order of the image of a plane of $V_{4}^{2}$ is always the order of the involution. Moreover it is not true that planes of opposite families on $V_{4}^{2}$ necessarily have images of the same order. For instance in a paper in this Bulletin [4] Clarkson discussed a simple involution of order two in which bundles of lines are transformed into $(3,1)$ congruences while plane fields of lines are transformed into $(1,1)$ congruences. In other words, on $V_{4}^{2}$ an $\omega$-plane is transformed into a surface of the fourth order, while a $\rho$-plane is transformed into a quadric surface.

Received by the editors May 10, 1948.

${ }^{1}$ Numbers in brackets refer to the bibliography at the end of the paper. 
Suppose, then, that the image of a general plane, $\pi$, on $V_{4}^{2}$ is a surface of order $r$. A general $S_{4}$ of $S_{5}$ will meet this image surface in a $C_{r}$ which must be the proper image of some curve in $\pi$, say a $C_{s}$. Since the order of the involution is two, the total image of a $C_{s}$ in $\pi$ is a $C_{2 s}$. Hence $r \leqq 2 s$. Now the $S_{4}$ which cuts out $C_{r}$ meets the image conic of any line, $l$, in $\pi$ in exactly two points, and these are necessarily the images of the points common to $C_{s}$ and $l$. Thus $C_{s}$ is met by any line in $\pi$ in two points. Hence its order is $s=2$ and so $r \leqq 4 .^{2}$

If the order of the image surface is four it clearly must lie properly in at least four dimensions, since the total intersection of $V_{4}^{2}$ and an $S_{3}$ is only a quadric surface. Consider first the possibility that it lies in a four-dimensional subspace of $S_{5}$. Then any $S_{3}$ in the $S_{4}$ which contains the image surface intersects the latter in a $C_{4}$, which, since the order of involution is two, must be the image of some curve in $\pi$ of order $s>1$. Now any line in $\pi$ will meet such a $C_{s}$ in $s$ points whose images will be points common to the conic into which the line is transformed, and the $C_{4}$. If $s>2$, the $S_{3}$ which cuts the $C_{4}$ will then contain the image conic of every line in $\pi$, which is absurd. Hence $s=2$. However, a general conic in $\pi$ meets the invariant locus in two points, whereas the $C_{4}$ cut by a general $S_{3}$ can meet it only once. The hyperplane sections of the image surface must therefore be the images of the conics through some invariant point which is simultaneously a singular point possessing a curve of images. Now on the $C_{4}$ cut from the image surface of $\pi$ by a general $S_{3}$ consider four coplanar points such that none of them is an image of the singular invariant point. Each of the $S_{3}$ 's of the pencil on the plane of these four points will cut the image surface in a $C_{4}$ which is the image of some conic through the singular invariant point. We thus have the impossible situation of $\infty^{1}$ conics passing through five fixed points, and so we must reject the possibility that the image of $\pi$ is a quartic surface in an $S_{4}$.

If the image of $\pi$ is a $V_{2}^{4}$ lying properly in $S_{5}$ its $\infty^{5}$ hyperplane sections must be the images of the $\infty^{5}$ conics in $\pi$. Each such curve must therefore have two invariant points, that is, must meet the invariant line in $\pi$ in two points. But this requires every $S_{4}$ in $S_{5}$ to pass through the invariant line in $\pi$, which is absurd. This, then, overthrows completely the possibility that the image of $\pi$ is of the fourth order.

Suppose next that the image of $\pi$ is a cubic surface, of necessity lying properly in some $S_{4}$, or more specifically, lying on the $V_{3}^{2}$ in which some $S_{4}$ meets $V_{4}^{2}$. Now by a well known theorem of Felix

2 This proof may be restated almost word for word for the general involution of order $m$, leading to the conclusion that the order of the image surface of a general plane of $V_{4}^{2}$ is not greater than $m^{2}$. 
Klein, any $V_{n-2}^{m}$ on a nonsingular $V_{n-1}^{2}$ in $S_{n}(n>3)$ is necessarily the complete intersection of $V_{n-1}^{2}$ with a single primal. In the present case we have a $V_{2}^{3}$ lying on a $V_{3}^{2}$ in $S_{4}$. Since a $V_{2}^{3}$ cannot be the complete intersection of a $V_{3}^{2}$ and any primal, it follows that the $V_{3}^{2}$ must be singular, that is, that it must be cut from $V_{4}^{2}$ by a tangent $S_{4}$. The cubic surface itself is cut from the singular $V_{3}^{2}$ common to $V_{4}^{2}$ and the tangent $S_{4}$ by a second hypercone having an entire plane in common with the first. All planes on the singular $V_{3}^{2}$ which belong to the same family as this common plane will meet the cubic surface in conics passing through the vertex of the $V_{3}^{2}$, and these conics will be the images of the lines of some pencil in $\pi$, since they have no free intersections among themselves, and since individually they have but one intersection with the image-conic of a general line of $\pi$. Now consider any line in the plane of one of these conics, $C$. Its image is a conic, $K$, which necessarily meets $\pi$ in two points on the line, $l$, whose image is $C$. The plane of the conic $K$ is thus a plane of $V_{4}^{2}$ meeting $\pi$ in $l$. But there is a unique plane of $V_{4}^{2}$ meeting a given plane in a given line. Hence the proper image of the plane of $C$ is the plane of $K$. Similarly all planes on the singular $V_{3}^{2}$ which belong to the same family as the plane of $C$ have for their images planes meeting $\pi$ in the lines of a certain pencil. In the aggregate these planes constitute the singular $V_{3}^{2}$ cut from $V_{4}^{2}$ by the $S_{4}$ which is tangent at the vertex of this pencil. Thus associated with the general plane, $\pi$, of at least one of the two families of planes on $V_{4}^{2}$ there are two singular $V_{3}^{2}$ s which are images of each other in such a way that the planes of one family on the first correspond respectively to the planes of one of the families on the second. But when all planes, $\pi$, are considered it is apparent that there must be $\infty^{2}$ such singular $V_{3}^{2}$ 's, collectively embracing $\infty^{3}$ planes which have only planes for their images. In other words if the planes of one family on $V_{4}^{2}$ have cubic surfaces for their images, the planes of the opposite family have planes for their images. But this is self-contradictory, because any plane and its image must meet in a line, since every plane contains a line of invariant points, and they must therefore belong to opposite families on $V_{4}^{2}$. Since the transformation is involutorial this requires that the planes of one family be simultaneously transformed into planes and into cubic surfaces, which is absurd.

Finally suppose that the images of all planes on $V_{4}^{2}$ are planes. Since a general line in a plane, $\pi$, is transformed into a conic on the image surface of $\pi$, it follows that any plane of $V_{4}^{2}$ meeting $\pi$ in a line has for its image a plane with a conic in common with the image of $\pi$. In other words any plane meeting $\pi$ in a line has an image which coincides with the image of $\pi$, which is impossible. This leaves a quadric 
surface as the only possibility for the image of a general plane of $V_{4}^{2}$.

Knowing now that the image of a general plane, $\pi$, is a quadric surface meeting $\pi$ in a line, consider a general line, $l$, in $\pi$. Its image is a conic on the image quadric of $\pi$, and this conic is met in two points by the image of any other line, $l^{\prime}$, in $\pi$. One of these points is of course the image of the intersection of $l$ and $l^{\prime}$. The other point must be in some sense a singular point, that is, it must be the common image of two distinct points in $\pi$. If as $l^{\prime}$ varied over $\pi$ this singular intersection varied along the image of $l$ it would follow that every point on the image of a general line was the image of more than one point. This contradicts the involutorial character of the transformation, and so necessarily the image conics of all lines in $\pi$ pass through a fixed point, $P$, on the quadric which is the image of $\pi$. Moreover since each line in $\pi$ contains a single point having $P$ for its image, the locus of such points in $\pi$ is a straight line.

Consider next the quadratic regulus formed by the rays joining the points of a general line, $l$, in $\pi$ to their respective images. Among the generators of the other family is one, $\lambda$, which passes through $P$ and which necessarily is met by every ray of the first family. For a second line, $l^{\prime}$, in $\pi$ there is similarly a line $\lambda^{\prime}$ which passes through $P$ and which is met by the image ray of every point on $l^{\prime}$. The image ray associated with the intersection, $Q$, of $l$ and $l^{\prime}$ thus meets both $\lambda$ and $\lambda^{\prime}$. Finally consider any third line, $l^{\prime \prime}$, of $\pi$. Its image regulus of course contains $P$. Moreover it contains the image ray of the intersection, $Q^{\prime}$, of $l$ and $l^{\prime \prime}$, and the image ray of the intersection, $Q^{\prime \prime}$, of $l^{\prime}$ and $l^{\prime \prime}$. The $S_{3}$ in which the image regulus of $l^{\prime \prime}$ lies thus contains both $\lambda$ and $\lambda^{\prime}$. In addition to $Q^{\prime}$ and $Q^{\prime \prime}$ it therefore contains $Q$, that is, it contains the entire plane $\pi$, which is absurd. The only escape is for $\lambda$ and $\lambda^{\prime}$ to coincide, which leads to the conclusion that there is one and only one line, $\lambda$, which is met by the image rays of every point of $\pi$. The uniqueness of $\lambda$ is evident, because if there were a second line (necessarily not passing through $P$ ) which was met by every image ray from $\pi$, it would determine with $\lambda$ an $S_{3}$ in which the image regulus of every line in $\pi$ would have to lie, which is clearly impossible.

Now let $R$ be the second intersection of $\lambda$ and $V_{4}^{2}$. For any line in $\pi$ some image ray must pass through $R$. Hence if such an image ray does not lie entirely on $V_{4}^{2}$ the image of every line in $\pi$ passes through $R$ as well as $P$, which is impossible. Thus the points of $\pi$ whose image rays pass through $R$ are the singular points in $\pi$, and the totality of the associated singular rays is the plane of $V_{4}^{2}, \sigma$, through $R$ and the 
line of singular points in $\pi$.

Consider now any other plane, $\pi^{\prime}$, on $V_{4}^{2}$ which meets $\pi$ in a line. As in $\pi$, the lines in $\pi^{\prime}$ map into conics on the image surface of $\pi^{\prime}$, and these conics all pass through a fixed point, $P^{\prime}$. Moreover the image ray of every point in $\pi^{\prime}$ meets a unique line, $\lambda^{\prime}$, through $P^{\prime}$, and this line intersects in a point, $R^{\prime}$, the singular ray, $s$, through the unique singular point, $S$, on the intersection of $\pi$ and $\pi^{\prime}$. And just as for $\pi$, all image rays of $\pi^{\prime}$ passing through $R^{\prime}$ are singular and in the aggregate form a plane, $\sigma^{\prime}$, of $V_{4}^{2}$ through $s$ and the line of singular points in $\pi^{\prime}$.

Any other line in $\pi$ through $S$ has a unique plane, $\pi^{\prime \prime}$, passing through it, and by the same reasoning as before, the singular rays through its line of singular points form a plane through $s$. But only two planes of $V_{4}^{2}$ pass through a line such as $s$. Hence this third plane cannot be distinct from both $\sigma$ and $\sigma^{\prime}$, and in fact must coincide with the plane of singular rays from $\pi^{\prime}$. This means that the singular points in $\pi^{\prime \prime}$ constitute a line of $\sigma^{\prime}$, which as $\pi^{\prime \prime}$ is allowed to vary covers all of $\sigma^{\prime}$. In other words the points of all singular rays from $\pi^{\prime}$ are themselves singular. Of course the same remark is true for the singular rays in $\sigma$.

Since there are at least two planes, $\sigma$ and $\sigma^{\prime}$, of singular points it follows that the linear complex of singular elements is special, that is, is tangent to $V_{4}^{2}$ at some point of $s$. But the reasoning leading to this conclusion could equally well have applied to any other singular ray, leading to the conclusion that the vertex of the singular complex had to lie upon it. Hence we infer that all singular rays are concurrent in the vertex of the singular complex. In other words $R$ and $R^{\prime}$, and the corresponding points associated with all the other planes of $V_{4}^{2}$, must coincide. Hence the lines $\lambda, \lambda^{\prime}, \cdots$ must coincide also. Thus we draw the conclusion that the image rays of all points on $V_{4}^{2}$ meet a fixed line, $\lambda$, through the vertex of the singular complex.

Consider again the image regulus of a general line $l$. The quadric of which it forms one family of generators is met by the $S_{4}$ which contains the singular complex in the singular ray, $s$, associated with $l$, and in a second line, $m$, which necessarily belongs to the same regulus as $l$. The image rays of all points of $l$ thus meet $m$, the points of $m$ and the image rays being in 1:1 correspondence. Moreover the image rays of all the points on the quadric common to $V_{4}^{2}$ and the $S_{3}$ determined by $\lambda$ and $l$ also meet $m$. For consider any generator of this quadric, $l^{\prime}$, belonging to the regulus not containing $l$. Like any other line on $V_{4}^{2}$ its image rays meet the singular $S_{4}$ in the points of some line, $m^{\prime}$. But such a generator meets $l$ in a point and the image 
conic of $l$ in a point, and these points are not images one of the other since their join, namely $l^{\prime}$ itself, is a line of $V_{4}^{2}$, which is impossible for a general pair of corresponding points. Thus the image rays of these points are distinct, and hence their intersections with $S_{4}$ are two distinct points of $m$. Therefore the line $m^{\prime}$ in which the image rays of a general generator, $l^{\prime}$, meet $S_{4}$ must coincide with $m$.

It is now easy to see that the image rays of the points of a general plane, $\pi$, meet $S_{4}$ in the points of some plane, $\sigma$. For if $l_{1}$ and $l_{2}$ be two general lines of $\pi$ meeting in a point $Q$, the image rays of each meet $S_{4}$ along a line. The two lines, $m_{1}$ and $m_{2}$, say, necessarily intersect, for otherwise the image ray of $Q$, which must meet both, would lie entirely in $S_{4}$, which cannot happen. Finally any other line, $l_{3}$, in $\pi$ meets $m_{1}$ and $m_{2}$, and so the line, $m_{3}$, along which its image rays intersect $S_{4}$ must meet $m_{1}$ and $m_{2}$, that is, must lie in the plane of $m_{1}$ and $m_{2}$.

Now at a general point, $P$, of $V_{4}^{2}$ consider three lines, $r_{1}, r_{2}$, and $r_{3}$ of $V_{4}^{2}$, so chosen that no one lies in the $S_{3}$ determined by $\lambda$ and either of the others. Each of the $S_{3}$ 's determined by $\lambda$ and one of the lines $r_{1}, r_{2}, r_{3}$ has the property noted above that the image rays of all the points which it has in common with $V_{4}^{2}$ meet $S_{4}$ along a line. Moreover these three lines, $m_{1}, m_{2}, m_{3}$, say, necessarily concurrent, are independent, that is define a specific $S_{3}$ in the singular $S_{4}$. Now any plane, $\pi$, on $V_{4}^{2}$ has the property that it meets any quadric on $V_{4}^{2}$ in at least one point. Hence the plane, $\sigma$, in which the image rays of $\pi$ meet $S_{4}$ must have one point on each of the three lines $m_{1}, m_{2}, m_{3}$, and these three points will all be distinct since $\pi$ does not in general intersect the conic cut from $V_{4}^{2}$ by the plane of $P$ and $\lambda$. Therefore $\sigma$ is contained entirely in the $S_{3}$ determined in $S_{4}$ by $m_{1}, m_{2}, m_{3}$. Thus in addition to meeting a fixed line, $\lambda$, through the vertex of the singular complex, the image ray of a general point of $V_{4}^{2}$ meets a fixed $S_{3}$ in the $S_{4}$ which cuts the singular complex from $V_{4}^{2}$. This completes our proof.

\section{BIBLIOGRAPHY}

1. C. R. Wylie, $A$ new series of line involutions, Accepted for publication in the Mathematics Magazine.

2. G. Schaake, Koninklijke Akademie van Wetenschappen te Amsterdam vol. 34 (1924-1925) pp. 478-485.

3. C. R. Wylie, $A$ series of line involutions in $S_{3}$ defined as point transformations of of a $V_{4}^{2}$ in $S_{5}$ into itself, National Mathematics Magazine vol. 14 (1939) pp. 125-133.

4. J. M. Clarkson, Some involutorial line transformations, Bull. Amer. Math. Soc. vol. (1933) pp. 149-154.

The University of Utah 